\title{
Species diversity and biting activity of Anopheles dirus and Anopheles baimaii (Diptera: Culicidae) in a malaria prone area of western Thailand
}

\author{
Chatchai Tananchai ${ }^{1 \dagger}$, Rungarun Tisgratog ${ }^{1 \dagger}$, Waraporn Juntarajumnong ${ }^{1 \dagger}$, John P Grieco ${ }^{2 \dagger}$, Sylvie Manguin ${ }^{3+}$, \\ Atchariya Prabaripai ${ }^{4 \dagger}$ and Theeraphap Chareonviriyaphap ${ }^{1 *+}$
}

\begin{abstract}
Background: A survey of adult anopheline mosquito diversities, collected from September 2009 to August 2010, was conducted in a malaria endemic area of western Thailand. Two anopheline species complexes, Dirus and Minimus, along with the Maculatus group were observed. Of several species documented from within each complex and group, four important malaria vectors were identified, including An. dirus, An. baimaii, An. minimus, and An. sawadwongporni. Information on biting activity and host preference for any single species within the Dirus complex has never been assessed. Using specific molecular identification assays, the trophic behavior and biting activity of each sibling species within the Dirus complex were observed and analyzed for the Kanchanaburi Province, Thailand.

Methods: Adult female mosquitoes were collected for two consecutive nights each month during a one year period. Three collection methods, human landing indoor (HLI), human landing outdoor (HLO), and cattle baited collections (CBC) were applied. Each team of collectors captured mosquitoes between 1800 and $0600 \mathrm{~h}$.

Results: From a total of 9,824 specimens, 656 belong to the Dirus complex (An. dirus 6.09\% and An. baimaii 0.59\%), 8,802 to the Minimus complex (An. minimus 4.95\% and An. harrisoni 84.65\%) and 366 to the Maculatus group (An. maculatus 2.43\% and An. sawadwongporni 1.29\%). Both An. dirus and An. baimaii demonstrated exophagic and zoophilic behaviors. Significantly greater numbers of An. dirus and An. baimaii were collected from cattle as compared to humans ( $P=0.003$ for $A n$. dirus and $P=0.048$ for An. baimaii).

Conclusions: Significantly greater numbers of An. dirus and An. baimaii were collected from cattle baited traps as compared to human landing collections $(P<0.05)$, demonstrating that both species show a strong zoophilic behavior. Knowledge of host-seeking behavior helps to define a species' capacity to acquire and transmit malaria and its contribution to the overall risk for disease transmission in the human population, as well as, assisting in the design and implementation of appropriate vector prevention and control strategies.
\end{abstract}

Keywords: Anopheles dirus, Anopheles baimaii, Exophagic, Zoophilic, Thailand

\section{Background}

In Thailand, malaria remains one of the most important infectious diseases despite years of well-organized disease control in reducing both mortality and morbidity countrywide [1]. Seventy percent of the malaria cases are documented from the relatively undeveloped borders and hill

\footnotetext{
* Correspondence: faasthc@ku.ac.th

${ }^{\dagger}$ Equal contributors

'Department of Entomology, Faculty of Agriculture, Kasetsart University, Bangkok 10900, Thailand

Full list of author information is available at the end of the article
}

region of eastern Myanmar, whereas the three species complexes, i.e. Anopheles dirus, An. minimus, and An. maculatus are commonly present and some of them are considered as important malaria vectors, including $A n$. dirus, An. baimaii, An. maculatus, An. sawadwongporni and An. minimus [2,3].

Better understanding of the behavior of each sibling species within the complex is quite important to help identify their respective roles in disease transmission and to assist the vector control personnel in designing the appropriate steps for vector control management.

\section{Biomed Central}


Due to some complexity within the $A n$. dirus complex, the closely related species cannot be differentiated from each other by morphological characters [4-9]. Some species are regarded as excellent malaria vectors because their anthropophagic nature brings them into frequent contact with humans, maintain high parasite loads, and exhibit a highly endophagic behavior [10,11]. Although various studies on the biting patterns and host preference of these complexes have been described in Thailand [12-17], most observations of mosquito biology and behaviors were based exclusively on the populations in which identification was made by morphological characters, except for one study on the An. minimus complex recently carried out by Sungvornyothin et al. [9]. Such morphological identification of the sibling species within a complex is not reliable and can lead to a high degree of misidentification $[9,18]$. The application of molecular techniques has made it possible to reliably identify species from entomological surveys. For the An. dirus complex, such molecular methods include an allele-specific polymerase chain reaction (AS-PCR), a sequence characterized amplified region (SCAR) and a rapid polymerase chain reaction, which are all considered useful tools for identifying the species within a complex $[8,19,20]$. In this study, we identified the species within the three complexes in the sympatric area of Pu Teuy Village, Sai Yok District, Kanchanaburi Province. This area is considered to be a malaria prone region with all three complexes responsible for malaria transmission being documented $[21,22]$. By using a molecular identification assay we were able to observe the trophic behavior, biting activity, and seasonal abundance in each sibling species within the $A n$. dirus complex, one of the most important complexes for malaria transmission in Thailand.

\section{Methods \\ Study site \\ Anopheles dirus was collected from the Pu Teuy Village, a village located in Sai Yok District, Kanchanaburi Province, western Thailand $\left(14^{\circ} 17^{\prime} \mathrm{N}, 99^{\circ} 11^{\prime} \mathrm{E}\right)$. The col- lection site is located approximately $1 \mathrm{~km}$ away from the village. It is surrounded by deep forest lying at approxi- mately $400 \mathrm{~m}$ above sea level. Local residents and their occupations are associated with the forest. A narrow, slow running stream ( $2 \mathrm{~m}$ wide and an average of $0.5 \mathrm{~m}$ deep) bordered with native vegetation runs across the village. Surrounding vegetation is primarily agricultural land used for growing papaya, cassava and maize and secondarily forest [17].}

\section{Collection methods}

Adult female mosquitoes were collected for two consecutive nights each month during a one year period. Three collection methods, including human landing indoor
(HLI), human landing outdoor (HLO), and cattle bait collections $(\mathrm{CBC})$ were utilized. Human landing collections were performed in an existing house with all windows closed and one door remaining open throughout the collection period. The entomological team was divided into two groups of four persons each. Two people collected mosquitoes inside the house, whereas the other two people collected outside. The distance between indoor and outdoor collections was set at $100 \mathrm{~m}$. The first team collected from 1800 to $2400 \mathrm{~h}$, followed by the second team that started at midnight and ended at $0600 \mathrm{~h}$. Both teams rotated their starting position between the first and second halves of the evening on each subsequent collection night to avoid collector bias. Collectors also rotated each night between indoor and outdoor locations. Human landing collections occurred uninterrupted for $45 \mathrm{~min}$ each hour. Cattle baited collections were conducted by a separate team of two collectors for $15 \mathrm{~min}$ each hour [17]. The $\mathrm{CBC}$ involved placing a single cow under the untreated cotton bed net, measuring $3.6 \mathrm{~m} \times 3.3 \mathrm{~m} \times 2.0 \mathrm{~m}$ (L:W: $\mathrm{H}$ ) with the net suspended $30 \mathrm{~cm}$ above the ground level to allow any hungry mosquitoes to get inside. The same cow was used throughout the study and was placed at least $50 \mathrm{~m}$ from the nearest human landing collection site but at equal distances from the forest fringe to avoid a potential distance bias in attracting mosquitoes. All collected mosquitoes were placed in clean, chemical free plastic cups that were labeled by hour and type of collection. Formal human-use approval was granted by the Ethical Research Committee convened by the Research and Development Institute, Kasetsart University, Thailand (KURDI-1/2553-1421457). All collected mosquitoes were held in netting covered plastic cups and were provided with a cotton pad soaked with $10 \%$ sugar solution. Each cup was returned to a local processing station in the field for morphological identification. Ambient air temperature and relative humidity were recorded from indoor and outdoor locations and at the site of the cattle baited trap each hour of the collection using a manual thermohygrometer (BARICO GmbH, Villingen-Schwenningen, Germany). Daily rainfall data was also recorded at the study site using a manual Rain Gauge (RAIN GAUGE AUGE KIT, England).

\section{Morphological and molecular species identification An. dirus complex}

Members of the An. dirus complex were identified to species using illustrated morphological keys for the adult Anopheles of Thailand [2,23]. In brief, female mosquitoes with a presector dark spot (PSD) on the radius vein that extended basally beyond the PSD spot on the costa vein and a PSD spot that reached the humeral dark (HD) spot of the costa vein or at least surpassed the middle of 


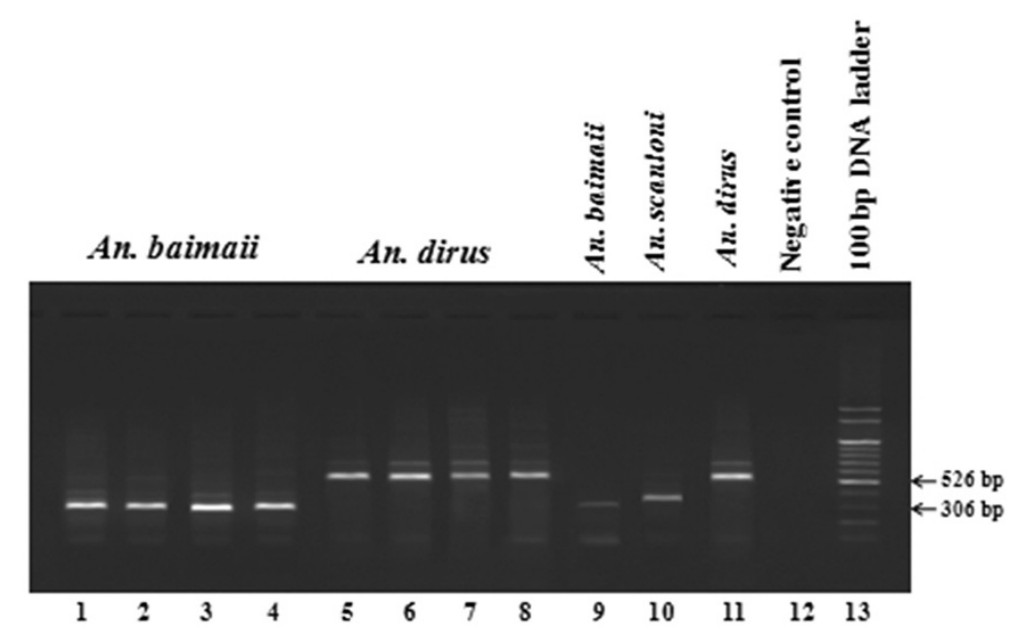

Figure 1 Multiplex Allele-Specific PCR assay. Lanes 1-4: An. baimaii; lanes 5-8: An. dirus; lanes 9-11: positive of An. baimaii, An. scanloni, An. dirus; lane 12: negative control; lane 13: 100 bp molecular ladder.

the presector pale spot (PSP) of the costa vein of at least one wing were identified as An. dirus. Molecular identifications using the AS-PCR assay of Walton et al. [8] and Audtho et al. [24] were subsequently applied to confirm the species identification, using the specific primers for Anopheles dirus, Anopheles cracens, Anopheles scanloni, Anopheles baimaii and Anopheles nemophilous (Figure 1).

\section{An. minimus complex}

Members of the An. minimus subgroup were identified to species using illustrated morphological keys for adult Anopheles in Thailand [2]. An. minimus was identified when a presector pale spot (PSP) was present on the costal vein of both wings, whereas $A n$. harrisoni required, at least, the presence of both presector pale and humeral pale spots (HP) on one wing [25]. Molecular identifications were performed using the AS-PCR assay of Garros et al. [26], and was conducted using primers specific for An. minimus and An. harrisoni.

\section{An. maculatus group}

All specimens of the An. maculatus group were identified to species using illustrated morphological keys for adult Anopheles in Thailand [2]. An. maculatus has the $\mathrm{R} 2+3$ wing veins with one dark spot on both wings and the presector dark (PSD) spot on the $\mathrm{R}$ vein is usually shorter than the PSD spots on the subcosta and costa. The R $4+5$ wing veins have 2 dark spots and the abdominal terga segments VII, VIII sometimes on VI are covered with dark scales on the posterolateral corners. Molecular identifications were performed using the AS-PCR assay of Walton et al. [27], and were conducted using primers specific for An. maculatus and $A n$. sawadwongporni.

\section{DNA extraction}

DNA for all Anopheles specimens was extracted using Genomic DNA from individual adult mosquitoes, according to the procedures of Linton et al. [28] and Manguin et al. [20].

\section{Data analysis}

Three key factors were selected for analysis and included seasonality, biting time and collection method. Seasonal periods were identified as wet (June to October), dry (November to February) and hot (March to May), biting times are separated into early evening (1800-2100 hours), late night (2100-2400 h), predawn (2400-0300 h) and dawn (0300-0600 h), and collection types are listed as indoor HLC, outdoor HLC and cattle baited captures.

The nocturnal biting cycle of $A n$. dirus and An. baimaii were tabulated by averaging the number of Anopheles landing per hour per human by indoor and outdoor location and by averaging the number of mosquitoes captured per cow per hour. Comparisons of landing data were analyzed by non-parametric Kruskal-Wallis tests. The accepted level of significance was determined at $0.05 \%(P$-valve $<0.05)$, followed by correlation coefficient $(r)$ analysis taking into account the correlation between mosquitoes captured and environmental variables. All data were analyzed using the SPSS statistical package (version 17.0, SPSS, Chicago, IL).

\section{Results}

Observations on adult anopheline diversity, collected from September 2009 to August 2010 at Pu Teuy Village, Kanchanaburi Province, western Thailand are given in Table 1. From a total of 9,824 specimens, 656 belong to the An. dirus complex (6.68\%), 8,802 were from the $A n$. minimus complex (89.6\%) and 366 were from the $A n$. 
Table 1 Monthly frequency of Anopheles mosquitoes at Pu Teuy Village, Sai Yok District, Kanchanaburi Province, for one year (September 2009-August 2010)

\begin{tabular}{|c|c|c|c|c|c|c|c|}
\hline \multirow[t]{3}{*}{ Month } & \multicolumn{7}{|c|}{ Anopheles complexes } \\
\hline & \multicolumn{2}{|c|}{ An. dirus } & \multicolumn{2}{|c|}{ An. minimus } & \multicolumn{2}{|c|}{ An. maculatus } & \multirow[t]{2}{*}{ Total } \\
\hline & DIR & BAI & MIN & HAR & MAC & SAW & \\
\hline September & 30 & 3 & 9 & 372 & 0 & 0 & 414 \\
\hline October & 54 & 9 & 7 & 234 & 0 & 0 & 304 \\
\hline November & 23 & 4 & 35 & 939 & 0 & 0 & 1001 \\
\hline December & 0 & 1 & 21 & 676 & 0 & 0 & 698 \\
\hline January & 0 & 0 & 65 & 824 & 0 & 0 & 889 \\
\hline February & 0 & 0 & 64 & 1115 & 0 & 0 & 1179 \\
\hline March & 0 & 0 & 34 & 713 & 4 & 0 & 751 \\
\hline April & 4 & 0 & 31 & 554 & 26 & 8 & 623 \\
\hline May & 17 & 4 & 43 & 873 & 43 & 30 & 1001 \\
\hline June & 51 & 5 & 89 & 783 & 43 & 30 & 1001 \\
\hline July & 124 & 13 & 74 & 994 & 77 & 42 & 1324 \\
\hline August & 295 & 19 & 14 & 239 & 50 & 35 & 652 \\
\hline Total & 598 & 58 & 486 & 8316 & 239 & 127 & 9824 \\
\hline
\end{tabular}

DIR: An. dirus, BAI: An. baimaii, MIN: An. minimus, HAR: An. harrisoni, MAC: An. maculatus, SAW: An. sawadwongporni.

maculatus group (3.72\%). Six important species were molecularly identified to include $A n$. dirus, An. baimaii, An. minimus, An. harrisoni, An. maculatus and $A n$. sawadwongporni (Table 1). Among these, four species, An. dirus, An. baimaii, An. minimus and An. maculatus are considered to be important malaria vectors in Thailand $[1,29]$. In the study, the two most recognized malaria vectors, $A n$. dirus and An. baimaii, were further characterized to obtain some bionomic information on the biting activity, host preference and density.

Table 2 provides the monthly distribution of $A n$. dirus and An. baimaii collected by the three collection methods during the one year study period. From a total of 656 specimens in the An. dirus complex, 598 specimens (91.2\%) were $A n$. dirus and 58 (8.8\%) were $A n$. baimaii. Greater numbers of these two species were collected during the rainy season (June to October) with a distinct peak in August. For An. dirus, 378 (63.2\%) were captured on cattle, 168 (28.1\%) were collected from outdoor human landing collection, and 52 (8.7\%) were obtained from indoor human landing collection. In contrast, a total of 58 specimens of $A n$. baimaii were captured from all three collection methods. Twenty-nine (50\%) were captured on cattle, 23 (39.7\%) were obtained from outdoor human landing collection, and 6 (10.3\%) from indoor human landing collection (Table 2). Our results showed that $A n$. dirus was more attracted by cattle than humans and, in the latter case, more outdoors than indoors, regardless of the time periods and climatic seasons.
Total mosquito biting frequencies by hour and collection method for An. dirus and An. baimaii are given in Figures 2-3. An. dirus specimens were found to exceed $A n$. baimaii in numbers for all collection periods. The indoor biting activity of An. dirus presented one prominent peak between 1900 and $2000 \mathrm{~h}$ and a smaller peak between 0200 and $0300 \mathrm{~h}$ (Figure 2). The outdoor human landing activity was elevated from 2300 to $2400 \mathrm{~h}$ (Figure 2). In contrast, cattle baited collections showed one clear peak for $A n$. dirus in the early evening (1900-2000 h) followed by a decline throughout the rest of the night (Figure 2). Although low numbers of $A n$. baimaii were obtained, the outdoor activity peaks were clearly defined with a distinct outdoor peak between 2400 and $0100 \mathrm{~h}$ whereas the indoor peak was difficult to determine due to the low number of specimens collected (Figure 3). Cattle baited catches showed two clear peaks for An. baimaii in the early evening (1900-2000 h) and midnight (2400-0100 h) (Figure 3).

The total number of landing mosquitoes per hour was subjected to a Kruskal-Wallis test, with seasons (dry, hot, and wet), collection methods (indoor and outdoor human bait and cattle bait) and time intervals (early evening, late evening, predawn, and dawn) as discriminating factors. A strong significant difference in the number of $A n$. dirus and $A n$. baimaii were found between seasons $\left(X^{2}=70.55 ; \mathrm{df}=2 ; P<0.0001\right.$ An. dirus and $\chi^{2}=27.34 ; \quad \mathrm{df}=2 ; \quad P<0.0001 \quad A n$. baimaii), and between indoor, outdoor human landing and cattle bait $\left(\chi^{2}=11.59 ; \mathrm{df}=2 ; P=0.003\right.$ An. dirus and $\chi^{2}=6.07 ; \mathrm{df}=2$; $P=0.048 A n$. baimaii). There was no significant difference in the number of either species collected between the four quarterly evening time intervals $\left(\chi^{2}=2.21 ; \mathrm{df}=3\right.$; $P=0.529 A n$. dirus and $\chi^{2}=3.68 ; \mathrm{df}=3 ; P=0.298 A n$. baimaii). Data from all collection methods was pooled to determine the correlation between mosquito abundance and environmental variables (Figure 4). An. dirus and $A n$. baimaii densities strongly correlated with the total rainfall $(r=0.454 ; P=0.016$ An. dirus and $r=0.609$; $P=0.003 A n$. baimaii) but were not related with relative humidity and minimum or maximum ambient air temperatures $(P>0.05)$.

\section{Discussion}

Of 73 known Anopheles species found in Thailand, some members of An. leucosphyrus and An. maculatus groups and $A n$. minimus subgroups, are recognized as important malaria vectors [2]. Five species have been incriminated as malaria vectors in Thailand, including $A n$. dirus [15], An. baimaii [30], An. minimus [31], An. pseudowillmori [30] and An. aconitus [30,32,33]. In this study, the six species found belong to two groups and one subgroup, including two members of the $A n$. dirus complex found in the $A n$. leucosphyrus group, $A n$. dirus and $A n$. 
Table 2 Total of monthly captures from three collection methods of Anopheles dirus and Anopheles baimaii from Pu Teuy Village, Sai Yok District, Kanchanaburi Province

\begin{tabular}{|c|c|c|c|c|c|c|c|c|c|c|c|}
\hline \multirow[t]{2}{*}{ Month } & \multicolumn{4}{|c|}{ An. dirus } & \multicolumn{4}{|c|}{ An. baimaii } & \multirow[t]{2}{*}{$\mathbf{T}$} & \multirow[t]{2}{*}{$\mathrm{H}$} & \multirow[t]{2}{*}{$\mathbf{R}$} \\
\hline & $\ln (\%)$ & Out(\%) & $\operatorname{Cow}(\%)$ & Total(\%) & $\ln (\%)$ & Out(\%) & $\operatorname{Cow}(\%)$ & Total(\%) & & & \\
\hline Sep & $7(23.3)$ & $9(30)$ & $14(46.7)$ & 30 & $0(0)$ & $0(0)$ & $3(100)$ & 3 & 28.97 & 78.38 & 168.70 \\
\hline Oct & $8(14.8)$ & $4(7.4)$ & $42(77.8)$ & 54 & $1(11.1)$ & $3(33.3)$ & $5(55.6)$ & 9 & 28.20 & 79.44 & 156.90 \\
\hline Nov & $0(0)$ & $5(21.7)$ & 18(78.3) & 23 & $1(25)$ & $3(75)$ & $0(0)$ & 4 & 20.08 & 85.44 & 156.90 \\
\hline Dec & $0(0)$ & $0(0)$ & $0(0)$ & 0 & $1(100)$ & $0(0)$ & $0(0)$ & 1 & 22.47 & 78.37 & 7.00 \\
\hline Jan & $0(0)$ & $0(0)$ & $0(0)$ & 0 & $0(0)$ & $0(0)$ & $0(0)$ & 0 & 20.37 & 83.47 & 12.10 \\
\hline Feb & $0(0)$ & $0(0)$ & $0(0)$ & 0 & $0(0)$ & $\mathrm{O}(0)$ & $0(0)$ & 0 & 26.11 & 77.63 & 0.00 \\
\hline Mar & $0(0)$ & $0(0)$ & $0(0)$ & 0 & $0(0)$ & $\mathrm{O}(0)$ & $0(0)$ & 0 & 25.63 & 83.06 & 76.50 \\
\hline Apr & $0(0)$ & $0(0)$ & $4(100)$ & 4 & $0(0)$ & $0(0)$ & $0(0)$ & 0 & 27.33 & 84.01 & 26.00 \\
\hline May & $2(11.8)$ & $3(17.6)$ & 12(70.6) & 17 & $1(25)$ & $2(50)$ & $1(25)$ & 4 & 26.36 & 83.11 & 63.00 \\
\hline Jun & $1(1.9)$ & $14(27.5)$ & $36(70.6)$ & 51 & $0(0)$ & $0(0)$ & $5(100)$ & 5 & 26.06 & 92.08 & 183.00 \\
\hline Jul & $12(9.7)$ & $50(40.3)$ & $62(50)$ & 124 & $1(7.6)$ & $6(46.2)$ & $6(46.2)$ & 13 & 24.86 & 88.83 & 291.00 \\
\hline Aug & $22(7.5)$ & $83(28.1)$ & $190(64.4)$ & 295 & $1(5.2)$ & $9(47.4)$ & $9(47.4)$ & 19 & 25.80 & 87.25 & 208.00 \\
\hline Total & 52 & 168 & 378 & 598 & 6 & 23 & 29 & 58 & & & \\
\hline (\%) & 8.7 & 28.1 & 63.2 & & 10.3 & 39.7 & 50.0 & & & & \\
\hline
\end{tabular}

R: Rainfall (mm), H: Humidity (\%), T: Temperature $\left({ }^{\circ} \mathrm{C}\right)$.

baimaii; two members of the An. maculatus group, An. maculatus and An. sawadwongporni; and two members of the $A n$. minimus complex belonging to the An. minimus subgroup, An. minimus and $A n$. harrisoni. Previous studies have successfully described the trophic behavior, biting activity, and seasonal abundance in the An. maculatus group and $A n$. minimus subgroup [9,34], whereas no such studies have documented these activities for the two most important malaria vectors within the $A n$. leucosphyrus group, An. dirus, An. baimaii.

Anopheles baimaii and An. dirus, are considered to be the primary malaria vectors in Thailand [2,35]. These two closely related species cannot be differentiated from each other by morphological characteristics alone $[8,25]$. Both are forest and forest-fringe mosquitoes that are considered highly anthropophilic [2,11]. The preferred

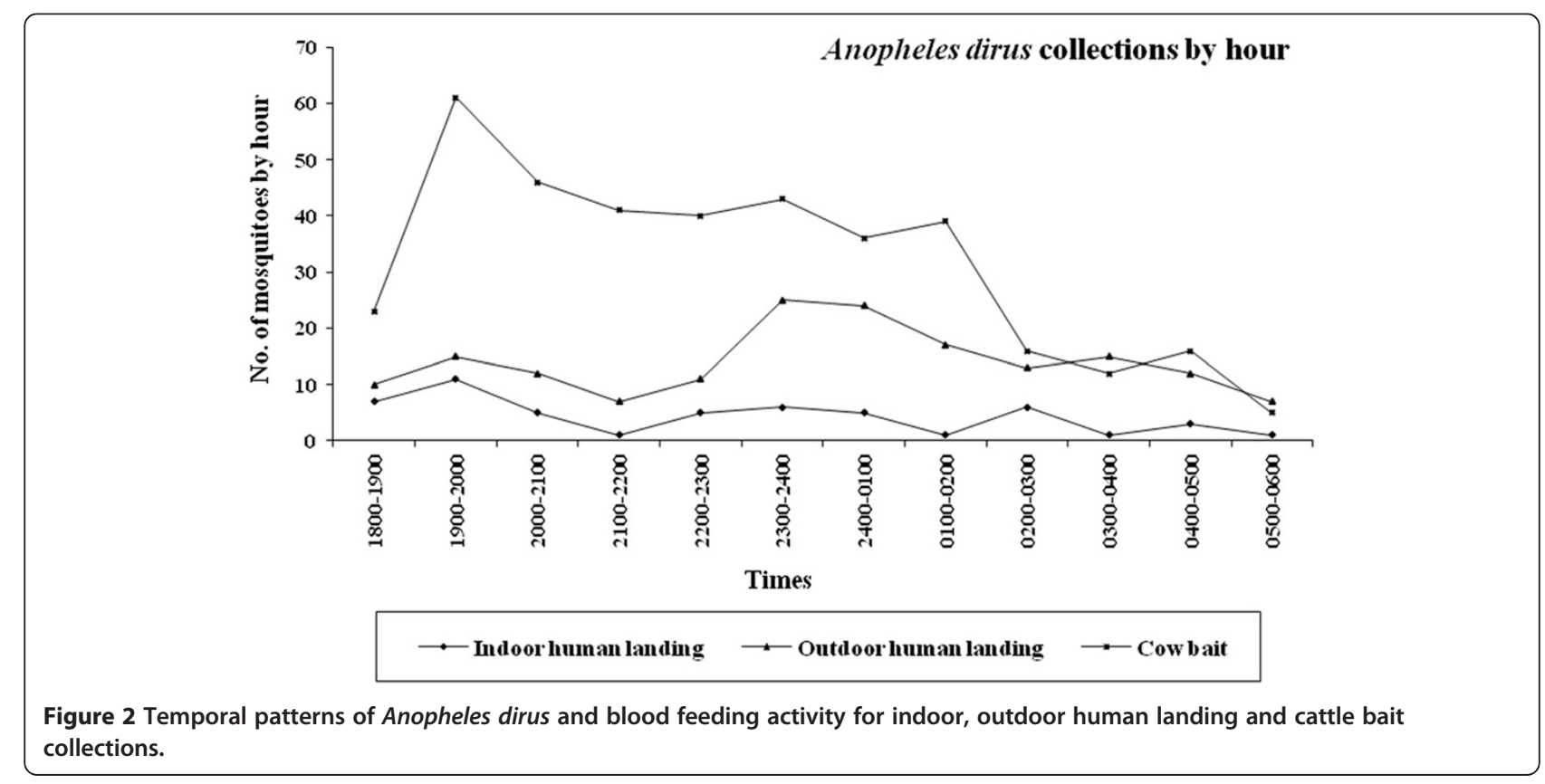




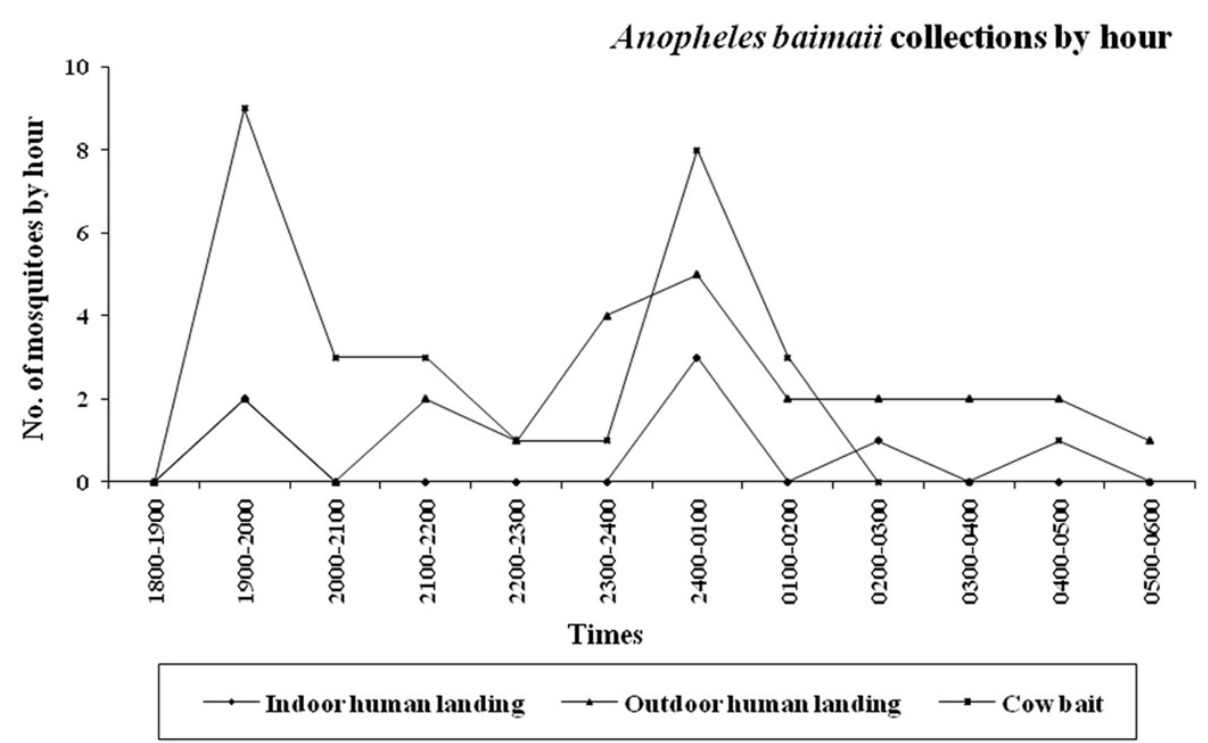

Figure 3 Temporal patterns of Anopheles baimaii blood feeding activity for indoor, outdoor human landing and cattle bait collections.

wheel-tracks and temporary ground pools in heavily shaded areas. In addition, larval habitats can be found in water jars, cut tree stumps, and root holes. An. dirus is the only species that is found throughout Thailand and often occurs in sympatry with An. baimaii in the western part of the country [36]. Both species are considered very anthropophilic in their blood feeding preference and demonstrate both exophagic and endophagic behaviors, and in some cases a generally greater tendency toward exophily $[3,5,35]$. However, interpretation of these early studies was hampered by species identification that was based on morphological characters, which was unreliable resulting in misidentification of species. This complicated the behavioral analysis of each sibling species within the complex $[3,17]$. Unlike these earlier studies, species of mosquitoes reported in this study were subjected to a multiplex AS-PCR, providing accurate species identification in order to describe with reliability the trophic behavior, seasonal abundance, and host preference of $A n$. dirus and An. baimaii in Pu Tuey Village, Kanchanaburi Province. An. dirus demonstrated a strong zoophilic behavior. More mosquitoes were caught on cattle bait

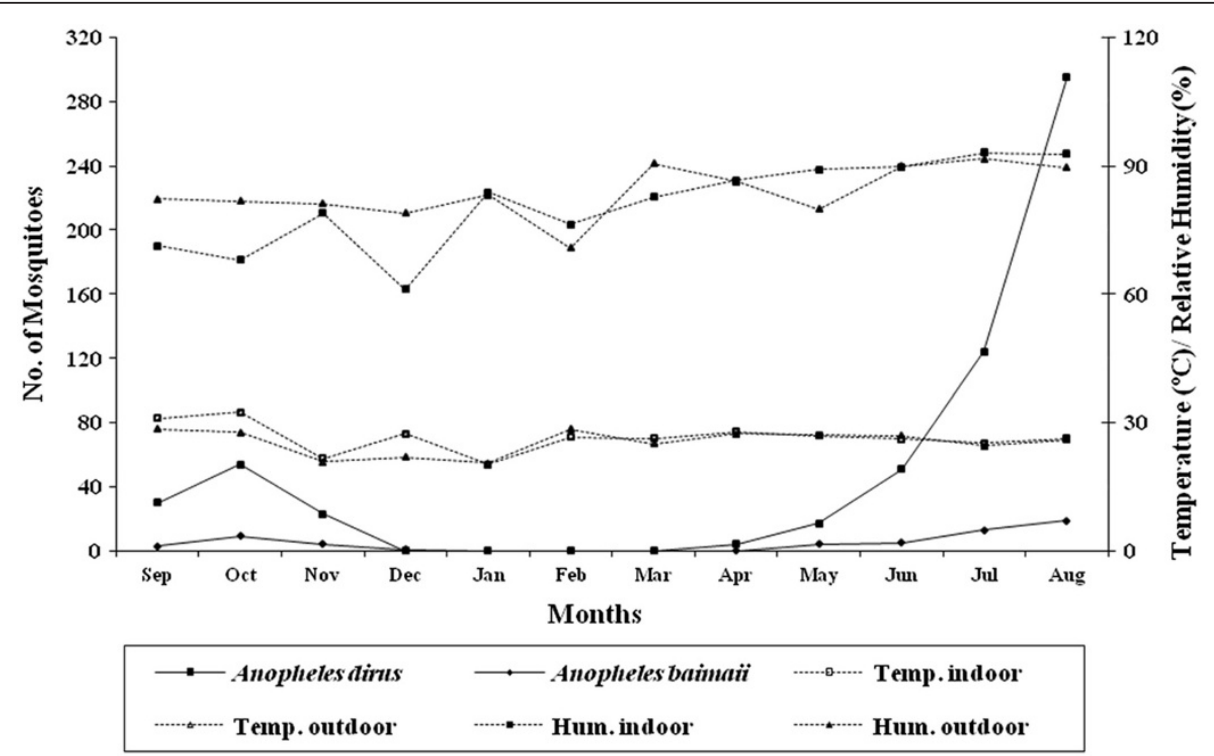

Figure 4 Monthly collections of Anopheles dirus and Anopheles baimaii in relation to average ambient air temperature and humidity in Pu Teuy Village, Sai Yok District, Kanchanaburi Province. 
compared to human landing from both inside and outside houses (63.2\% of $A n$. dirus). Results of previous studies on biting activity and host preference of $A n$. dirus complex in central Thailand demonstrated a delayed and more prolonged feeding peak that occurred between 2000 and $2400 \mathrm{~h}[13,14]$. In southern Thailand, Scanlon and Sandhinand [12] reported the peak biting activity to occur between 2400 and $0300 \mathrm{~h}$. Rosenberg et al. [15] observed a biting peak that occurred between 2200 and $0100 \mathrm{~h}$, and Rattanarithikul et al. [16] reported a single early-evening biting peak between 2000 and $2200 \mathrm{~h}$. In western Thailand, Sungvornyothin et al. [17] observed the biting activity between 2000 and $2100 \mathrm{~h}$ for both indoor, outdoor human landing collections and between 2000 and $2300 \mathrm{~h}$ in cattle baited collections. All of those biological and behavioral studies for An. dirus used only morphological characteristics for identification. This high degree of variability in biting patterns for populations found throughout Thailand might be the result of the mixed populations of species.

Our study described the biting behavior and blood feeding activities based on a clear and reliable identification of the sibling species of the An. dirus complex using PCR technology. The indoor biting activity of An. dirus recorded in this study demonstrated a peak between 1900 and $2000 \mathrm{~h}$. Outdoor human landing collections identified a prominent peak between 2300 and $0100 \mathrm{~h}$. On the other hand, cattle baited collections showed one clear peak in the early evening between 1900 and $2100 \mathrm{~h}$, followed by a declining trend. An. baimaii was collected in a small proportion compared to An. dirus and showed an ambiguous pattern of feeding activity. Indoor numbers were too small to evaluate but outdoor activity peaked between 2400 and $0100 \mathrm{~h}$. Cattle baited catches showed two clear peaks at both 1900-2100 h and at $2400-0100 \mathrm{~h}$. Accurate identification of the species using multiplex PCR technology could explain the differences in feeding patterns of these sibling species complexes when compared to earlier published reports. Previous studies have shown that the morphological identification alone of the two sibling species of the $A n$. minimus complex is not reliable and can result in nearly $40 \%$ misidentification of specimens $[9,18]$.

The seasonal abundance of the Anopheles mosquitoes collected in this study appeared to be influenced by several factors. Adult densities were found to be positively associated with increased rainfall (July to August). This is clearly seen from the fact that the greatest numbers of adults were found during the wettest period of the year. Rainfall dependent abundance patterns have previously been reported for Thailand and Bangladesh $[5,10,17,37]$. This suggests that high rainfall provides adequate larval habitats for An. dirus that prefers temporary breeding habitats such as animal footprints, wheel tracks, and temporary ground pools common during the wet season. An inverse relationship with rainfall was documented for $A n$. minimus [9] and $A n$. maculatus in the same locality [34], wherein these two species have been shown to prefer breeding at the edges of slow-running streams $[9,38]$. Heavy rainfall would flush these habitats out resulting in reduced adult densities. In contrast, a negative association was found with a higher mean ambient temperature and relative humidity. Although, the number of malaria cases is low in Pu Teuy Village, the area remains at risk for increased transmission due to a high degree of human movement (parasite introduction) from highly malarious areas. Also, the current findings agree with previous work that demonstrated that efficient malaria vectors such as, $A n$. dirus, An. minimus and An. maculatus are commonly found in greater abundance during the rainy periods of the year.

\section{Conclusions}

Accurate identification of the species with the use of multiplex PCR technology could explain the differences in feeding patterns of these sibling species, An. dirus and An. baimaii in Pu Teuy Village as compared to previously published work from other localities in Thailand. The current findings confirm that these species have a higher propensity to feed outdoors compared to indoors as previously published. A better understanding of the members of the Dirus sibling species complex, An. dirus and An. baimaii behavior related to host preference and both temporal and spatial feeding activity will help facilitate the design and efficiency of malaria vector control operations in Thailand.

\section{Competing interests}

The authors declare that they have no competing interests.

\section{Authors' contributions}

All the authors have contributed significantly to this study. TC and JPG assisted with study design. CT, RT and TC did the laboratory and field data collection. SM and WJ carry out the molecular genetic studies. AP performed statistical analysis. All authors contributed to the production of the manuscript and revision, with TC as final guarantor. All authors read and approved the final version of the manuscript.

\section{Acknowledgements}

We thank the Armed Forces Development Command, Sai Yok District, Kanchanaburi Province, Thailand, for permission to study areas. The Thailand Research Fund Organization (Senior Research Scholar Program: RTA528007) funded this research.

\section{Author details}

'Department of Entomology, Faculty of Agriculture, Kasetsart University, Bangkok 10900, Thailand. ${ }^{2}$ Department of Preventive Medicine and Biometrics, Uniformed Services University of Health Sciences, Bethesda, MD 20814, USA. ${ }^{3}$ Institut de Recherche pour le Développement (IRD), UMR-MD3, Montpellier, France. ${ }^{4}$ Department of Mathematics, Statistics and Computer, Faculty of Liberal Arts and Science, Kasetsart University, Kamphaengsean, Nakhonpathom 73140, Thailand.

Received: 1 August 2012 Accepted: 17 September 2012

Published: 25 September 2012 


\section{References}

1. Ministry of Public Health (MOPH): Annual report bureau of vector borne disease. Thailand: Department of Disease Control, Ministry of Public Health; 2010.

2. Rattanarithikul $R$, Harrison BA, Harbach RE, Panthusiri $P$, Coleman RE: Illustrated keys to the mosquitoes of Thailand. IV. Anopheles. Southeast Asian J Trop Med Public Health 2006, 37:1-128.

3. Manguin S, Garros C, Dusfour I, Harbach RE, Coosemans M: Bionomics, taxonomy, and distribution of the major malaria vector taxa of Anopheles subgenus Cellia in Southeast Asia: an updated review. Infect Genet Evol 2008, 8:489-503.

4. Baimai V: Population cytogenetics of the malaria vector Anopheles leucosphyrus group. Southeast Asian J Trop Med Public Health 1988, 19:667-680

5. Baimai V, Kijchalao U, Sawadwongporn P, Green CA: Geographic distribution and biting behaviour of four species of the Anopheles dirus complex (Diptera: Culicidae) in Thailand. Southeast Asian J Trop Med Public Health 1988, 9:151-161.

6. Sawadipanich Y, Baimai V, Harrison BA: Anopheles dirus species E: chromosomal and crossing evidence for another member of the Dirus complex. J Am Mosa Control Assoc 1990, 6:477-481.

7. Poopittayasataporn A, Baimai V: Polytene chromosome relationships of five species of the Anopheles dirus complex in Thailand. Genome 1995 38:426-434

8. Walton C, Handley JM, Kuvangkadilok C, Collins FH, Harbach RE, Baimai V, Butlin RK: Identification of five species of the Anopheles dirus complex from Thailand, using allele-specific polymerase chain reaction. Med Vet Entomol 1999, 13:24-32.

9. Sungvornyothin S, Muenvorn V, Garros C, Prabaripai A, Bangs MJ, Manguin S, Chareonviryaphap T: Trophic behavior and biting activity of the two sibling species of the Anopheles minimus complex in western Thailand. J Vector Ecol 2006, 31:252-261.

10. Rosenberg R, Maheswary NP: Forest malaria in Bangladesh. II. Transmission by Anopheles dirus. AmJTrop Med Hyg 1982, 31:183-191.

11. Baimai V, Green CA, Andre RG, Harrison BA, Peyton EL: Cytogenetic studies of some species complexes of Anopheles in Thailand and Southeast Asia. Southeast Asian J Trop Med Public Health 1984, 15:536-546.

12. Scanlon JE, Sandhinand J: The distribution and biology of Anopheles balabacensis in Thailand (Diptera: Culicidae). J Med Entomol 1965, 2:61-69.

13. Wilkinson RN, Miller TA, Esah S: Anthropophilic mosquitoes in central Thailand, with notes on Anopheles balabacensis Baisas and malaria. Mosa News 1970, 30:146-148.

14. Baimai V, Poopittayasataporn A, Kijchalao U: Cytological differences and chromosomal rearrangements in four members of the Anopheles dirus complex (Diptera: Culicidae). Genome 1988, 30:372-379.

15. Rosenberg R, Andre RG, Somchit L: Highly efficient dry season transmission of malaria in Thailand. Trans R Soc Trop Med Hyg 1990, 84:22-28.

16. Rattanarithikul R, Linthicum KJ, Konishi E: Seasonal abundance and parity rates of Anopheles species in southern Thailand. J Am Mosa Control AssoC 1996, 12:75-83.

17. Sungvornyothin S, Kongmee M, Muenvorn V, Polsomboon S, Bangs MJ, Prabaripai A, Tantakom S, Chareonviriyaphap T: Seasonal abundance and blood feeding activity of Anopheles dirus sensu lato in western Thailand. J Am Mosq Control Assoc 2009, 25:425-430.

18. Jaichapor B, Kengluecha A, Rongnoparut P, Rueda LM, Sithiprasasna R: Morphological variations of Anopheles minimus A in Tak province, Thailand. Southeast Asian J Trop Med Public Health 2005, 36:609-615.

19. Huong NT, Sonthayanon P, Ketterman AJ, Panyim S: A rapid polymerase chain reaction based method for identification of the Anopheles dirus sibling species. Southeast Asian J Trop Med Public Health 2001, 32:615-620.

20. Manguin $S$, Kengne $P$, Sonnier $L$, Harbach RE, Baimai $V$, Trung HD, Coosemans M: SCAR markers and multiplex PCR-based identification of isomorphic species in the Anopheles dirus complex in Southeast Asia. Med Vet Entomol 2002, 16:46-54.

21. Chareonviriyaphap T, Bangs MJ, Ratanatham S: Status of malaria in Thailand. Southeast Asian J Trop Med Public Health 2000, 31:225-237.

22. Kongmee M, Achee NL, Lerdthusnee K, Bangs MJ, Chowpongpang S, Prabaripai A, Chareonviriyaphap T: Seasonal abundance and distribution of Anopheles larvae in a riparian malaria endemic area of western Thailand. Southeast Asian J Trop Med Public Health 2012, 43:601-613.
23. Rattanarithikul R, Panthusiri P: Illustrated keys to the medically important mosquitoes of Thailand. Southeast Asian J Trop Med Public Health 1994, 25:1-66.

24. Audtho M, Tassanakajon A, Boonsaeng V, Tpiankijagum S, Panyim S: Simple nonradioactive DNA hybridization method for identification of sibling species of Anopheles dirus (Diptera: Culicidae) complex. J Med Entomol 1995, 32:107-111.

25. Sungvornyothin S, Garros C, Chareonviryaphap T, Manguin S: How reliable is the humeral pale spot for identification of cryptic species of the Minimus Complex? J Am Mosa Control Assoc 2006, 20:185-191.

26. Garros C, Koekemoer LL, Coetzee M, Coosemans M, Manguin S: A single multiplex assay to identify major malaria vectors within the African Anopheles funestus and the Oriental An. minimus groups. AmJTrop Med Hyg 2004, 70:583-590.

27. Walton C, Somboon P, O'Loughlin SM, Zhang S, Harbach RE, Linton YM, Chen B, Nolan K, Duong S, Fong MY, Vythilingam I, Mohammed ZD, Trung HD, Butlin RK: Genetic diversity and molecular identification of mosquito species in the Anopheles maculatus group using the ITS2 region of rDNA. Infect Genet Evol 2007, 7:93-102.

28. Linton YM, Harbach RE, Chang Moh S, Anthony TG, Matusop A: Morphological and molecular identity of Anopheles (Cellia) sundaicus (Diptera: Culicidae), the nominotypical member of a malaria vector species complex in Southeast Asia. Syst Entomol 2001, 26:357-366.

29. Manguin S, Bangs MJ, Pothikasikorn J, Chareonviriyaphap T: Review on global co-transmission of human Plasmodium species and Wuchereria bancrofti by Anopheles mosquitoes. Infect Genet Evol 2010, 10:159-177.

30. Green CA, Rattanarithikul R, Pongparit S, Sawadwongporn P, Baimai V: A newly recognized vector of human malarial parasites in the Oriental region, Anopheles (Cellia) pseudowillmori (Theobald, 1910). Trans $R$ Soc Trop Med Hyg 1991, 85:35-36.

31. Rattanarithikul R, Konishi E, Linthicum KJ: Detection of Plasmodium vivax and Plasmodium falciparum circumsporozoite antigen in anopheline mosquitoes collected in southern Thailand. AmJTrop Med Hyg 1996, 54:114-121

32. Gould DJ, Esah S, Pranith U: Relation of Anopheles aconitus to malaria transmission in the central plain of Thailand. Trans $R$ Soc Trop Med Hyg 1967, 61:441-442.

33. Maheswary NP, Khan Z, Molla FR, Haq MI: Incrimination of Anopheles annularis van der Wulp-1854 [sic] as an epidemic malaria vector in Bangladesh. Southeast Asian J Trop Med Public Health 1993, 24:776-778.

34. Muenworn V, Sungvornyothin S, Kongmee K, Polsomboon S, Bangs MJ, Akrathanakul P, Tanasinchayakul S, Prabaripai A, Chareonviriyaphap T: Biting activity and host preference of the malaria vectors Anopheles maculatus and Anopheles sawadwongporni (Diptera: Culicidae) in Thailand. J Vector Ecol 2008, 34:62-69.

35. Sinka ME, Bangs MJ, Manguin S, Chareonviriyaphap T, Patil AP, Temperley WH, Gething PW, Elyazar IRF, Kabaria CW, Harbach RE, Hay SI: The dominant Anopheles vectors of human malaria in the Asia-Pacific region: occurrence data, distribution maps and bionomic précis. Parasit Vectors 2011, 4:89.

36. Rattanarithikul R, Green CA, Panyim S, Noigamol C, Chanaimongkol S, Mahapibul P: Larval habitats of malaria vectors and other Anopheles mosquitoes around a transmission focus in northwestern Thailand. J Am Mosq Control Assoc 1995, 11:428-433.

37. Dutta P, Khan SA, Bhattarcharyya DR, Khan AM, Sharma CK, Mahanta J: Studies on the breeding habitats of the vector mosquito Anopheles baimai and its relationship to malaria incidence in Northeastern region of India. Breeding habitats of Anopheles baimai and its role in incidence of malaria in Northeastern region of India. EcoHealth 2010, 7:498-506.

38. Chareonviriyaphap T, Prabaripai A, Bangs MJ, Aum-Aung B: Seasonal abundance and blood feeding activity of Anopheles minimus Theobald (Diptera: Culicidae) in Thailand. J Med Entomol 2003, 40:876-881.

doi:10.1186/1756-3305-5-211

Cite this article as: Tananchai et al:: Species diversity and biting activity of Anopheles dirus and Anopheles baimaii (Diptera: Culicidae) in a malaria prone area of western Thailand. Parasites \& Vectors 2012 5:211. 